\title{
Effect of Heat and Mechanical Treatments on Mechanical Properties of Al-Zn-Mg-Cu Alloys
}

\author{
Fouad El Dahiye \\ Department of Design and Production, Mechanical and Electric Engineering Collage, Al- \\ University of Baath -Homs-Syria \\ Mahmoud AlAssaad \\ Department of Design and Production, Assistant Professor,Mechanical and Electric \\ Engineering Collage, University of Baath -Homs-Syria \\ Mohamad Yehea Alnefawy \\ Department of Design and Production, Mechanical and Electric Engineering Collage / Al- \\ University of Baath-Homs-Syria \\ Yahya.netawy@gmail.com
}

\begin{tabular}{|l|l|l|}
\hline Submission date:- 20/11/2018 & Acceptance date:- 3/1/2019 & Publication date:- 20/1/2019 \\
\hline
\end{tabular}

\begin{abstract}
In this research the effect of various heat treatments on tensile properties and hardness of Aluminum-Zinc-Magnesium-Copper alloy (7075 Aluminum alloy) was studied, with and without mechanical treatment by extrusion process. The 7075 alloy samples were submitted to two type of heat treatments, T6 heat treatment by artificial ageing samples in $120^{\circ} \mathrm{C}$ for $24 \mathrm{~h}$ and retrogression and reageing (RRA). Where in RRA treatment the first ageing done in $120^{\circ} \mathrm{C}$ for $24 \mathrm{~h}$, then the retrogression done in $180^{\circ} \mathrm{C}$ for $30 \mathrm{~min}$ and re-ageing in $120^{\circ} \mathrm{C}$ for $24 \mathrm{~h}$. We got the best results by apply the RRA treatment with extrusion process, where tensile strength was reached to $380 \mathrm{Mpa}$ without extrusion machining process, while reached to $530 \mathrm{Mpa}$ with extrusion process
\end{abstract}

Key words: Al-Zn-Mg-Cu alloy, Artificial aging, Retrogression and re-ageing, Mechanical treatment, Tensile Properties, Vickers Hardness. 


\section{Al-Zn-Mg-Cu تأثير المعالجات الحرارية و الميكانيكية على الخواص الميكانيكية لسبائك *** * محمد يحيى النيفاوي \\ محمود الأسعد * م \\ فؤ اد ضحية \\ " قسم هندسة التصميم و الإنتاج، كلية الهندة المبكانيكبة والكهربائية، جامعة البعث/سوريا \\ "* قسم هندسة التصميج والإنتاج، كلية العندسة المبكانيكبة والكهربائية، جامعة البعث/ سوربيا

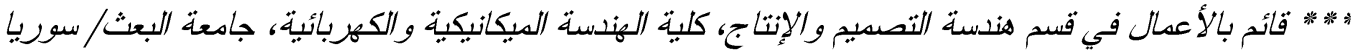

Yahya.netawy@gmail.com

الخلاصة

يدرس هذا البحث تأثير المعالجات الحرارية المختلفة على خواص الثند والقساوة لسبيكة ألمنيوم-زنك-مغنبزيوم-نحاس

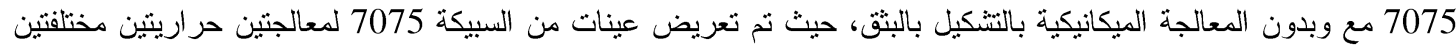

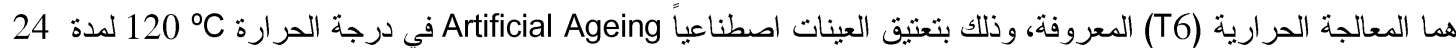
h، والمعالجة الحرارية الثانية هي الإرجاع و إعادة التعتيق Retrogression and re-ageing (RRA) حيث نم التعتيق الأول في

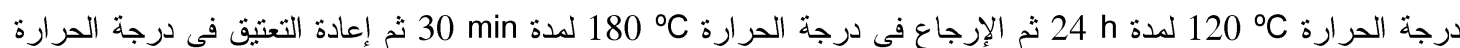
. $24 \mathrm{~h}$ h $120^{\circ} \mathrm{C}$

وقد حصلنا على أفضل النتائج بتطبيق المعالجة RRA مع عملية التثكيل بالبثق حيث بلغت مقاومة الثند 380 بدون Mpa بالثي عملية التشكيل بالبثق وبلغت 530 Mpa مع عملية التتكيل بالبثق. كلمات الداله: سيبكة ألمنيوم-زنك-مغنيزيومنحاس، التعتيق الاصطناعي، إرجاع و إعادة التعتيق، معالجات ميكانيكية، خواص الثند، قساوة فيكرز.

Introduction 1 المقدمة

ينتثر فلز الألمنيوم في صخور الأرض بنسبة تفوق كل الفلزات الأخرى، فهو يمثل نحو 8\% من القثرة الأرضية،

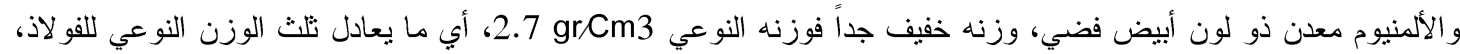

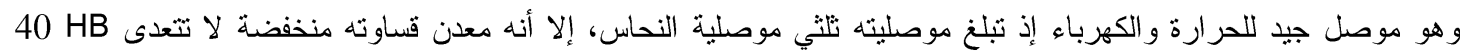

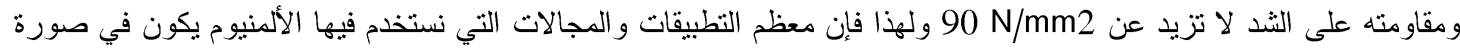
سبائكية بإضافة عناصر سبائكية للألمنيوم، و إن العناصر السبائكبة الأساسية التي تضاف للألمنيوم هي: النحاس Cu ، و و المغنيزيوم

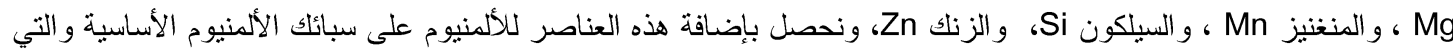

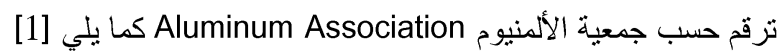

$$
\begin{aligned}
& \text { 1xxx } \\
& \text { 2xxx } \\
& \text { 3xxx } \\
& \text { 4xxx }
\end{aligned}
$$

تتميز كل سلسلة من هذه السلاسل بخاصية تميز ها عن باقي السلاسل كالموصلية الكهربائية المتازة التي تتمتع بها سبائك

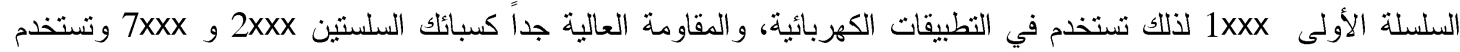

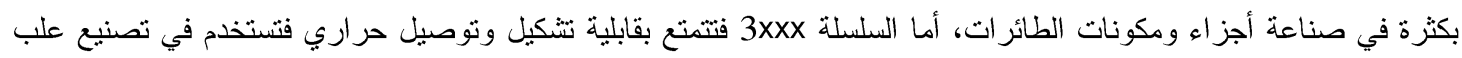


Journal of University of Babylon for Engineering Sciences, Vol. (27), No. (1): 2019.

المشروبات الغازية و المبادلات الحر ارية، ونتمتع سبائك السلسلة 5xxx بمقاومة ممتازة للتآكل لذلك تستخدم في صناعة السفن، أما

سبائك السلسة 6xxx فنتمتع بقابلية ممتازة للتتكيل بالبثق لذلك تصنع منها المنتجات المتعددة ذات المقاطع المختلفة بالبثق [2xx. درس العديد من الباحثين خو اص سبائك السلسلة 7xxx من سبائك الألمنيوم وهي سبائك ألمنيوم-زنك-مغنيزيوم-نحاس،

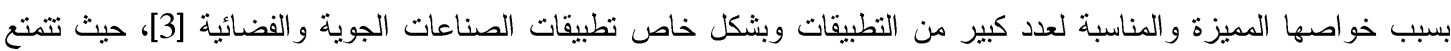
سبائك الألمنيوم عالية المقاومة 7xxx بخو اص ممتازة وخاصةً بعد تطبيق المعالجات الحرارية عليها كمقاومة شد عالية، وقابلية تثكيل ممتازة، ومقاومة ثآكل مقبولة، واحدة من أكثر هذه المعالجات الحرارية أهمية هي معالجات الإرجاع وإعادة التعنيق Retrogression and re-aging (RRA)

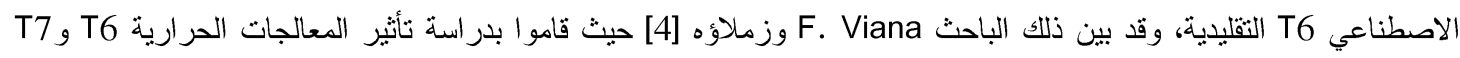

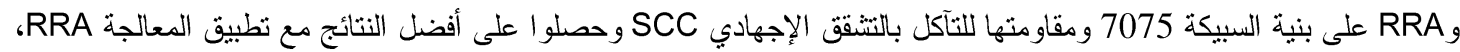
أما الباحث Adeyemi Dayo Isadare وزملاؤه [5] فقد قاموا بدراسة تأثير معالجة التلدين على خواص السبيكة 7075، 7075، وقام الباحث M.F. Ibrahim وزملاؤه [6] بدراسة تأثنير زمن المعالجة الحر ارية المحلولية على خواص السبيكة 7075.

وكذلك قام الباحث A.F. Oliveira وزملاؤه [7] بدر اسة تأثير معالجات RRA, T6 على مقاومة التآكل بالتشقق الإجهادي SCC و القساوة ومقاومة الثد للسبائك 7050 و7150 وكذلك أعطت المعالجة RRA أفضل النتائج. في حين درس الباحث

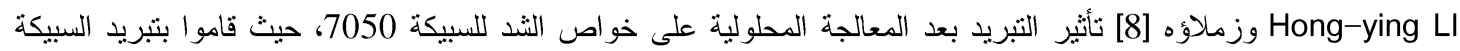
بعد المعالجة الحرارية المحلولية بدءاً من درجات حرارة مختلفة وحصلو ا على أعلى قيم لمقاومة الثند وإجهاد الخضوع عند السقاية بدءاً من الدرجة C C 450 و التي بلغت على التو الي 491 Mpa و 573 ـ

وقد درس الباحث Prasanta Kumar Rout وزملاؤه [9] نأثثر التعتيق ثثائي المراحل على خواص الثد والقساوة للسبيكة 7017. أما الباحث لبر CHEN Song-yi [10] وزملاؤه فقاموا بدر اسة تأثير التعتيق ثلاثي المراحل على متانة ومقاومة السبيكة 7085 للتآكل و لاحظو ازيادة مقاومة الثد في التعنيق الثثاثي بمقدار 5\% عن ثثائي المراحل مع تحسين في مقاومة التآكل بالتشقق الإجهادي.

ودرس الباحث M. Esmailian وزملاؤه [11] تأثير زمن معالجة الإرجاع على خو اص السبيكة 7055 ذلك في معالجة

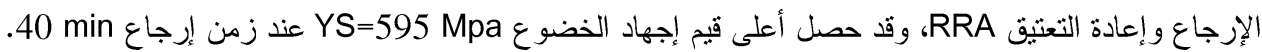
وقد لاحظنا عدم تطرق الباحثين-في الأبحاث المذكورة آنفاً-تنأثير المعالجة الميكانيكية (التشكيل) مع المعالجات الحرارية

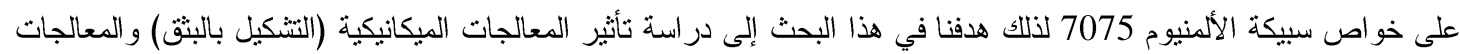

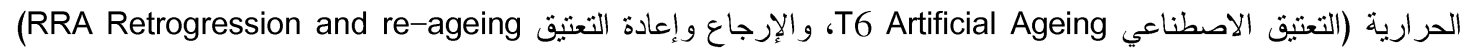
على الخو اص الميكانيكية (خو اص الثد والقساوة) لسبيكة ألمنيوم-زنك-مغنيزيوم-نحاس

Materials and Methods 2. المواد والأسياليب

نم استلام السبيكة 7075 موضوع البحث وكانت على شكل بيليت بسماكة [Cm] 12، ويبين الجدول 1 التركيب الكيميائي لسبيكة ألمنيوم -زنك -مغنيزيوم -نحاس 7075.

الجدول (1) التركيب الكيميائي لسبيكة ألمنيوم 7075.

\begin{tabular}{|c|c|c|c|c|c|c|c|}
\hline \multicolumn{8}{|c|}{ (\% wt) 7075 Th النسب الوزنية للعناصر في السبيكة } \\
\hline Si & Ti & Cr & Fe & Cu & Mg & Zn & Al \\
\hline 0.06 & 0.03 & 0.18 & 0.24 & 1.8 & 2.9 & 6.7 & Balance \\
\hline
\end{tabular}


تم صهر كمية من السيبكة 7075 في بوتثة غرافيتية في فرن كهربائي، وقد تمت عملية الصهر عند درجة حرارة

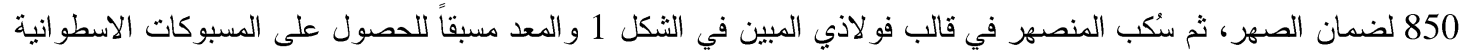

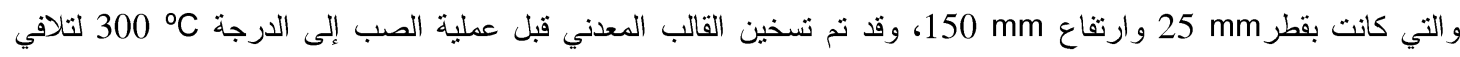

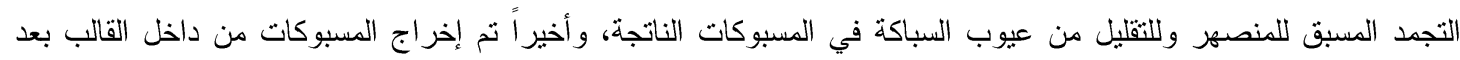

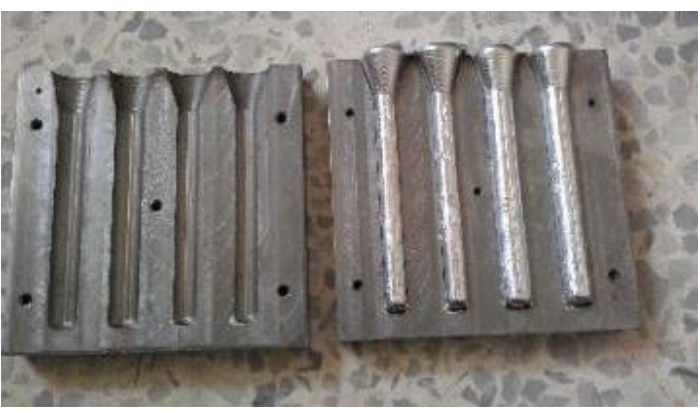
ثبريده.

الشكل 1 القالب الفولاذي المستخدم للصب .

عُرضت جميع المسبوكات للمعالجة الحرارية المحلولية في درجة الحرارة Co C 480 لددة 8 ف ثم تمت السقاية في الماء

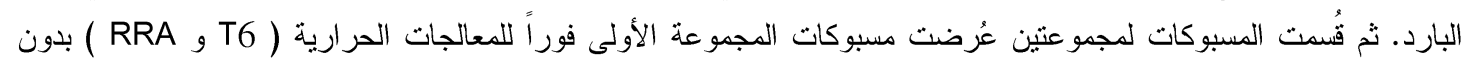

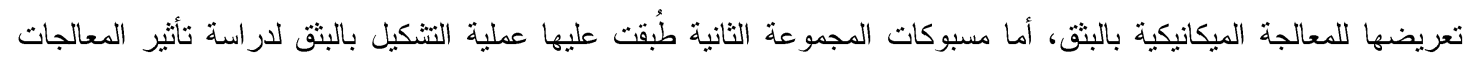

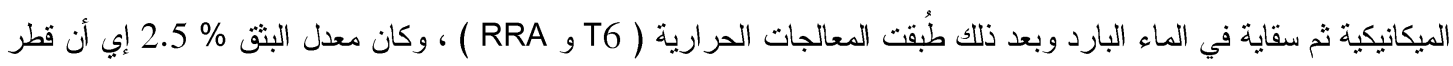

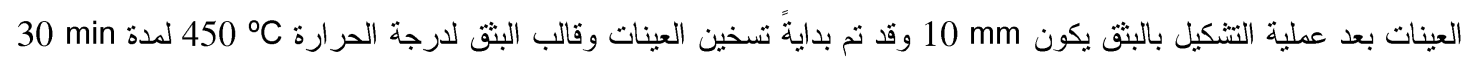

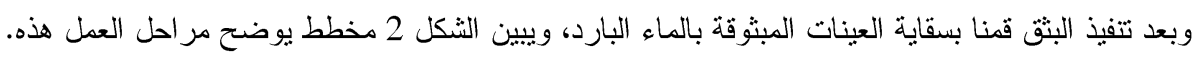

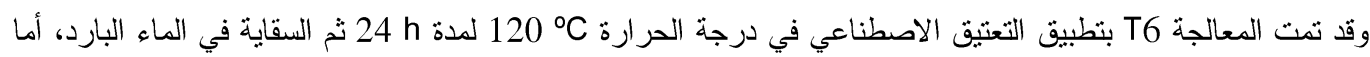

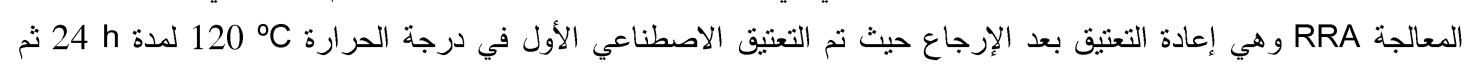

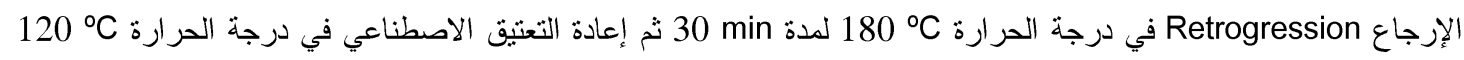
لمدة 24 24، ويبين الثنكل 3 الفرن الكهربائي المستخدم لتطبيق هذه المعالجات الحرارية. 
Journal of University of Babylon for Engineering Sciences, Vol. (27), No. (1): 2019.
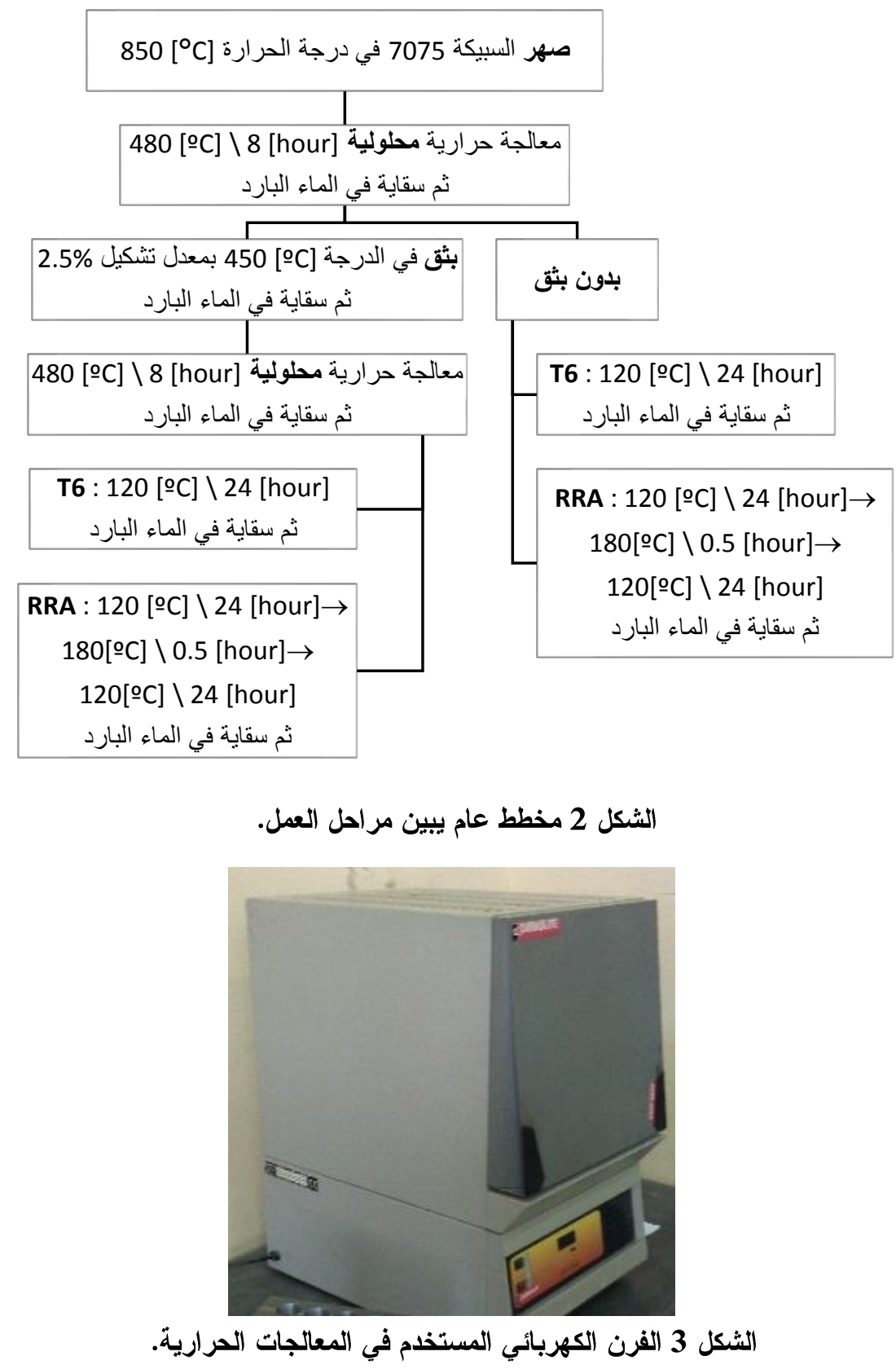

3esults and Discussion المناقشة و النتائج

يبين الجدول 2 مواصفات السيبكة 7075 فبل نعريضها لأي نوع من المعالجات الميكانيكية (التثكيل بالبثق) أو الحرارية

(RRA أو T6)

الجدول (2) مواصفات الميكانيكية للسبيكة 7075 قبل تعريضها لأي نوع من المعالجات.

\begin{tabular}{|c|c|c|}
\hline قساوة فيكرز قHV] & $\begin{array}{c}\text { إجهاد الخضوع [N/mm²] } \\
\text { [N/mm }\end{array}$ & 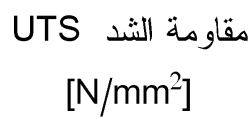 \\
\hline 100 & 160 & 180 \\
\hline
\end{tabular}


تم إجراء اختبار الثند وفق المواصفة ASTM-B557 عن طريق جهاز الاختبار TINIUS OLSEN H25KS المبين في

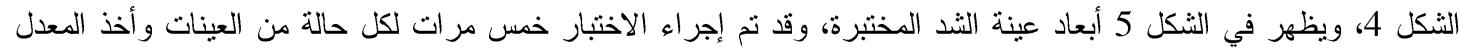

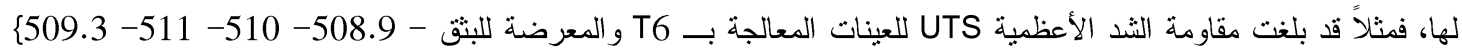
20 512.13 N/mm2\} فتكون القيمة المنوسطة لهذه القيم 510 N/mm2 تقريباً.

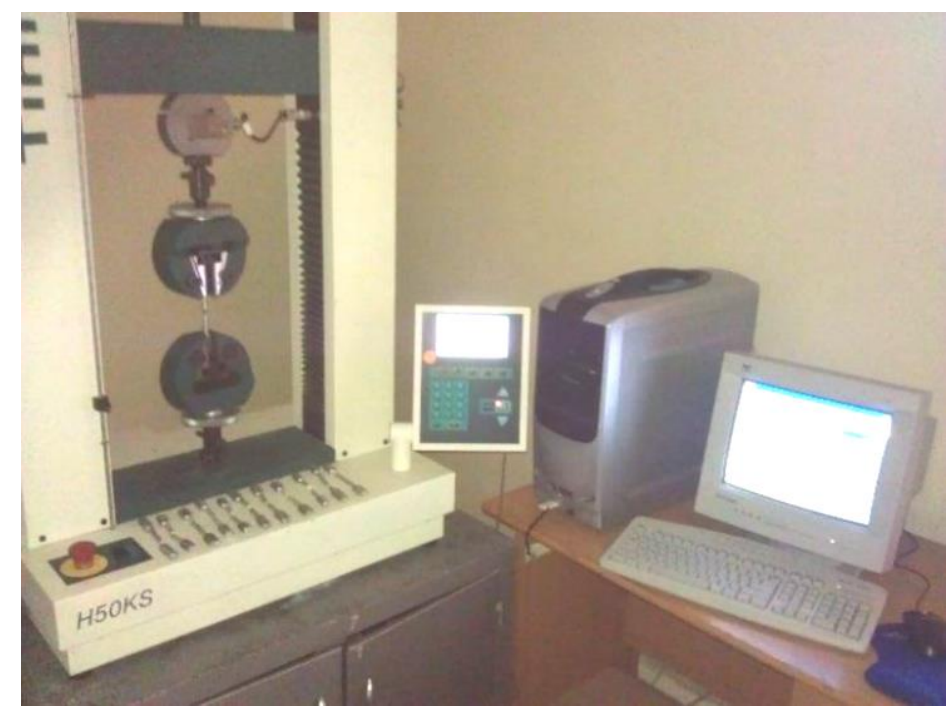

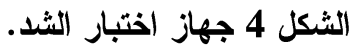

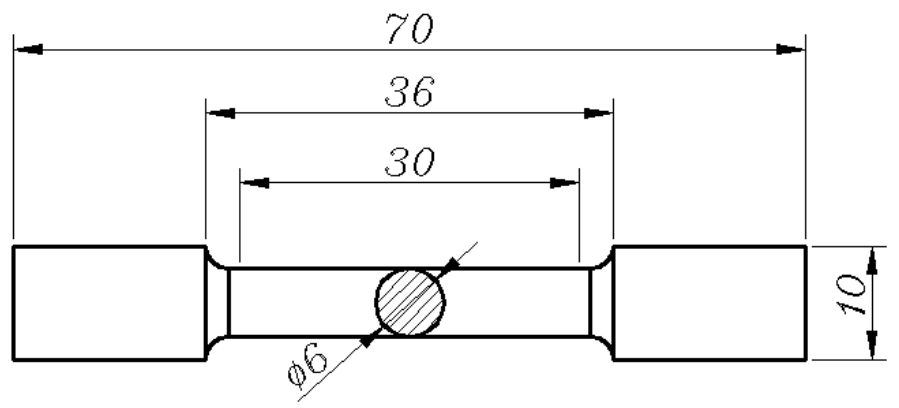

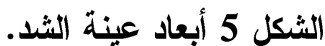

ويظهر في الثكل 6 و الثنكل 7 قيم إجهاد الخضوع Yield Strength (YS) ومقاومة الثند Uimate Tensile Strength (UTS) 
Journal of University of Babylon for Engineering Sciences, Vol. (27), No. (1): 2019.

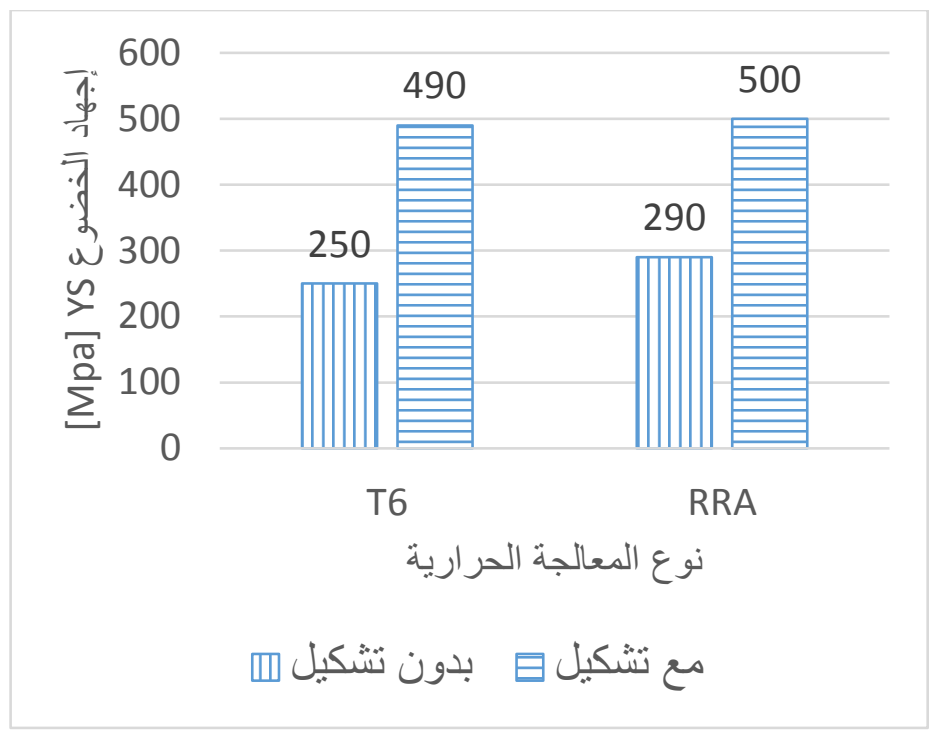

الثكل 6 قيم إجهاد الخضوع للسبيكة 7075 معالجة حرارياً بـ T6 وRRA قبل وبعد التشكيل.

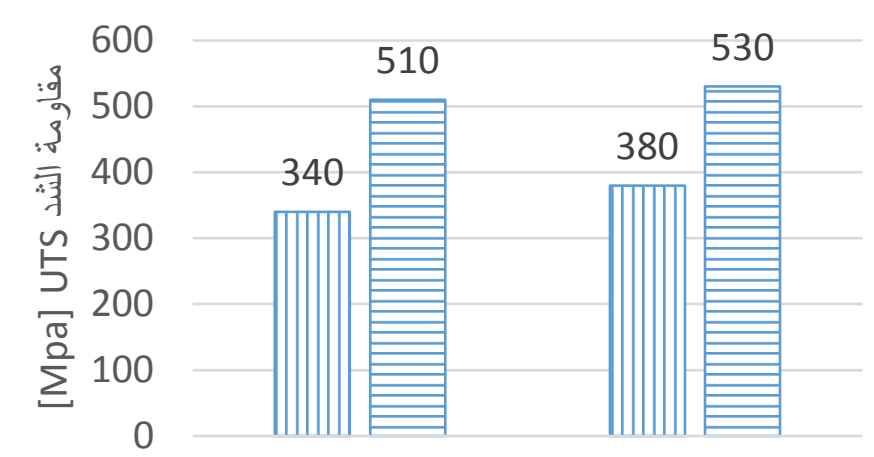

$$
\begin{aligned}
& \text { T6 نوع المعالجة الحرارية } \\
& \text { RRA }
\end{aligned}
$$

مع تشكيل

الثكل 7 قيم مقاومة الثد للسبيكة 7075 معالجة حرارياً بـ T6 و قبل وبعد التشكيل.

3.2. القساوة

تم إجراء اختبار القساوة وفق المواصفة ASTM-E92 بواسطة جهاز قياس قساوة فيكرز المبين في الثكل 8 وهو من

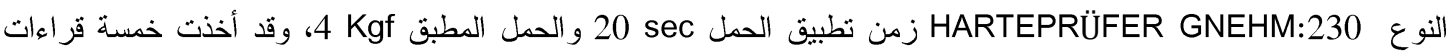
للقساوة من كلّ عينة من مناطق مختلفة لتحديد القيمة المتوسطة للقساوة. ويظهر في الثكل 9 قيم قساوة فيكرز للسبيكة 7075 معالجة حرارياً بـ RRA و6 قبل وبعد التشكيل 
Journal of University of Babylon for Engineering Sciences, Vol. (27), No. (1): 2019.

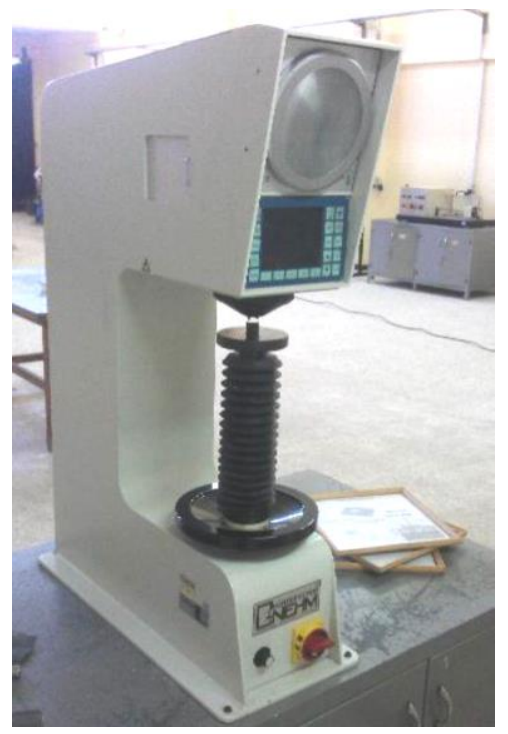

الثكل 8 جهاز قياس قساوة فيكزز.

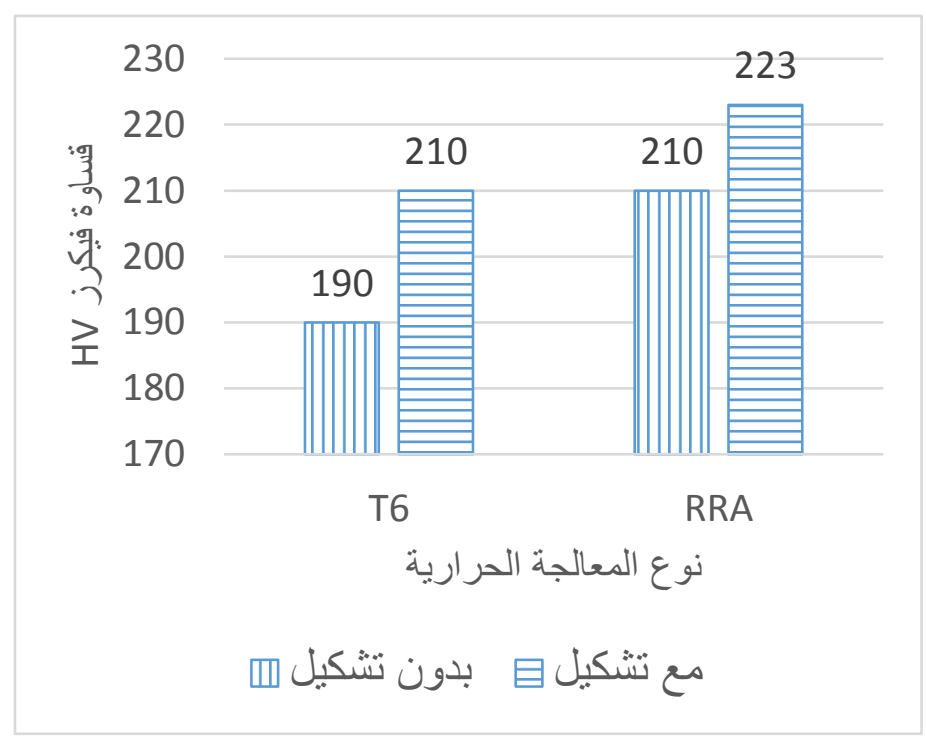

الثكل 9 قيم قساوة فيكرز للسبيكة 7075 معالجة حرارياً بـ T6 وRRA قبل وبعد النثكيل.

ونلاحظ من الأشكال 6 و7 و9 بأن خو اص الثد (YS, UTS) وقساوة فيكرز للسبيكة 7075 عند معالجتها بتطبيق معالجة الإرجاع و إعادة التعتيق RRA كانت ذات قيم أعلى مما كانت عليه عند معالجة نفس السبيكة بالمعالجة T6، حيث كانت قيم إجهاد الخضوع 250 Mpa ومقاومة الثند العظمى 340 Mpa و القساوة 190 ذللك عند تطبيق المعالجة T6، في حين بلغت هذه القيم لإجهاد الخضوع 290 Mpa ومقاومة الثند العظمى 380 Mpa و القساوة RRA 210 HV عند نطبيق معالجة و و إن جميع هذه القيم

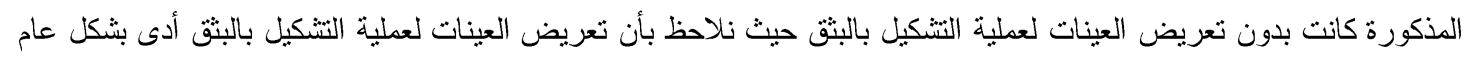
لتحسن جميع هذه الخواص لتصبح من أجل المعالجة T6 إجهاد الخضوع 490 Mpa ومقاومة الثد العظمى 510 و القساوة بعa 210 HV إجهاد الخضوع RRA ومن أجل المعالجة 500 Mpa ومقاومة الثد العظمى 530 و القساوة 223 HVa.

3.3 الصور المجهرية

للحصول على الصور المجهرية وفق المواصفة ASTM-E3 تم استخدم المجر الضوئي B-353 Met Optika وبمقدار تكبير 200X حيث شُحذت سطوح العينات باستخدام جهاز الثحذ نصف الآلي بورق الثحذ المائي ذات الدرجات 600، 800، 
Journal of University of Babylon for Engineering Sciences, Vol. (27), No. (1): 2019.

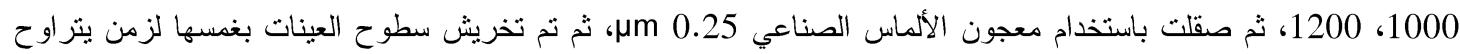
بين (20-10) ثانية. في محلول الإظهار Keller's Reagent وهو ذو التركيب النالي [1200]:

$\left\{1 \mathrm{Cm}^{3} \mathrm{HF}, 1.5 \mathrm{Cm}^{3} \mathrm{HCL}, 2.5 \mathrm{Cm}^{3} \mathrm{HNO}_{3}, 95 \mathrm{Cm}^{3} \mathrm{H}_{2} \mathrm{O}\right\}$

ويبين الثكل 10 الصور المجهرية لعينات مختلفة من السبيكة 7075 ويظهر فيه:

الثكل a-10 عينة من السيبكة 7075 قبل تعريضها للمعالجات الحرارية (T6 أو RRA) وبدون تعريضها للتتكيل بالبثق، وقد احتوت هذه العينة على تثعبات Dendrites أولية للمحلول الصلب الغني بالألمنيوم وشبكة النشعبات الداخلية والمكونات

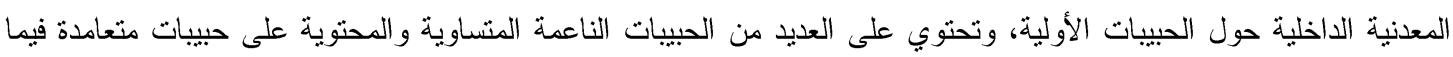

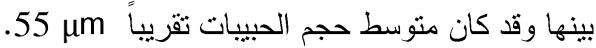

الثكل b-10 عينة من السبيكة 7075 معرضة للمعالجة الحرارية T6 قبل تعريضها للتشكيل بالبثق، بدأت كثافة الثبكة

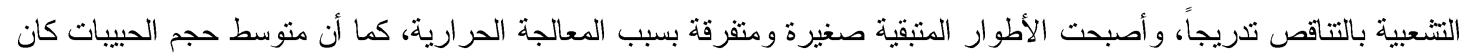

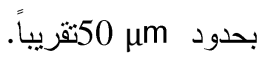

والثكل c-10 لعينة من السبيكة 7075 معرضة للمعالجة الحرارية RRA قبل تعريضها للتثكيل بالبثق، و أصبحت كثافة

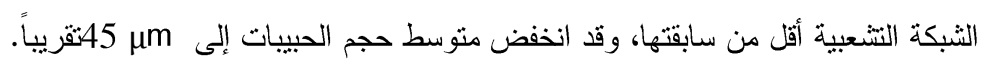

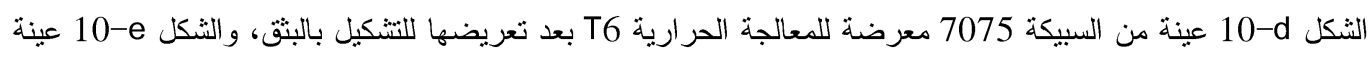
من السيبكة 7075 معرضة للمعالجة الحرارية RRA بعد تعريضها للتتكيل بالبثق ونلاحظ فيها تغير شكل الحبييات بسبب عملية النتكيل بالبثق.

ويمكن ملاحظة أن المعالجات الحرارية قد أدت لانخفاض الحجم الحيبي وتتعيم البنية البلورية للسيكة 7075 وأن

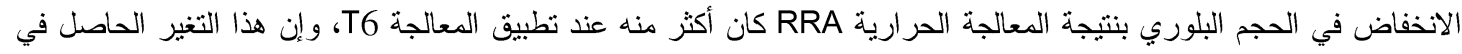

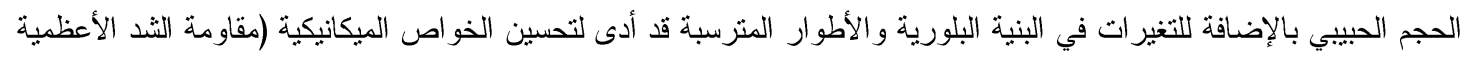

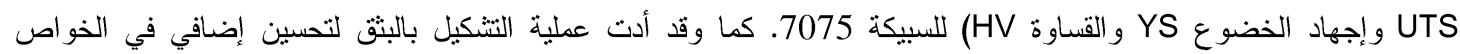
الميكانيكية للسبيكة 7075 بفعل التصليد الانفعالي.

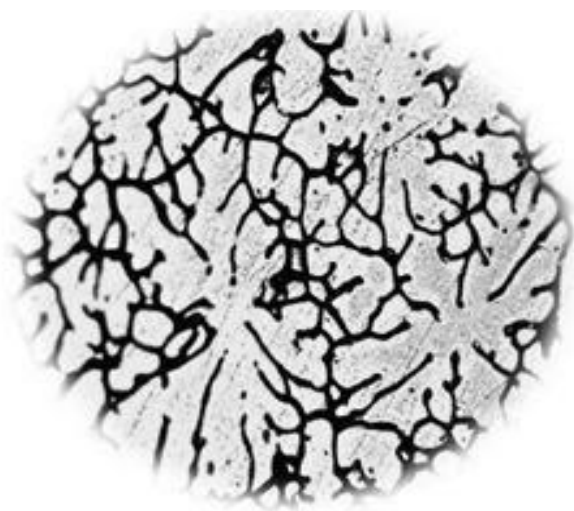

a 


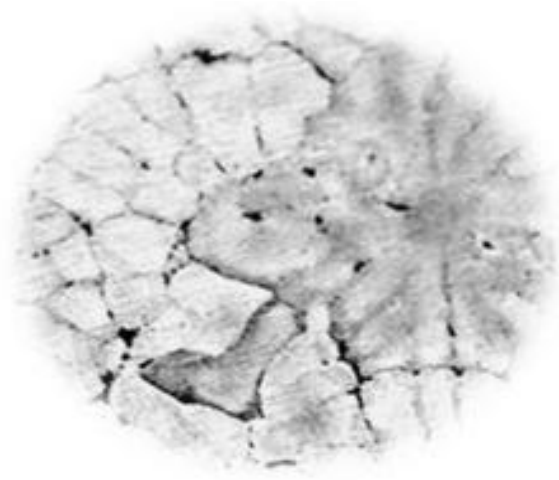

C

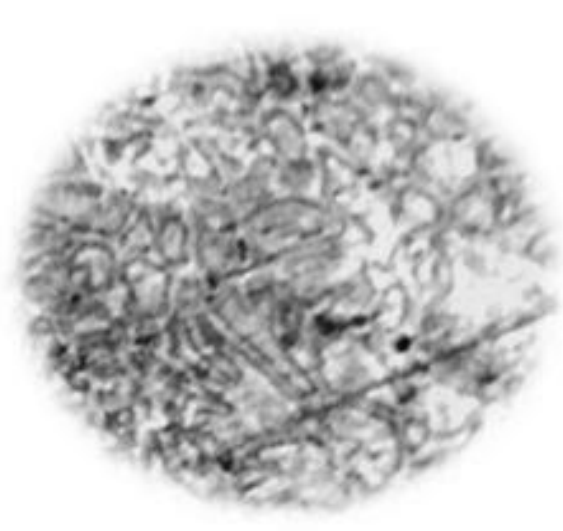

e

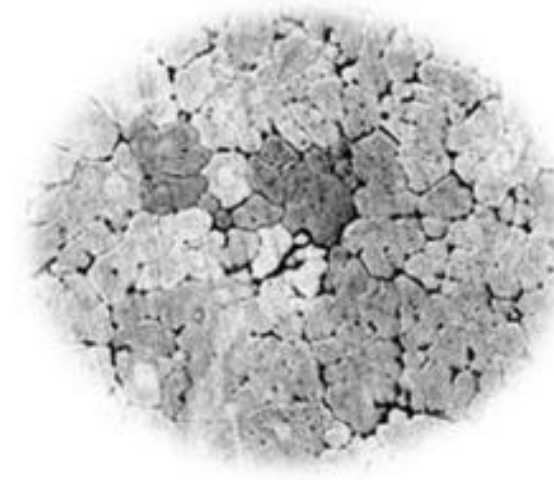

b

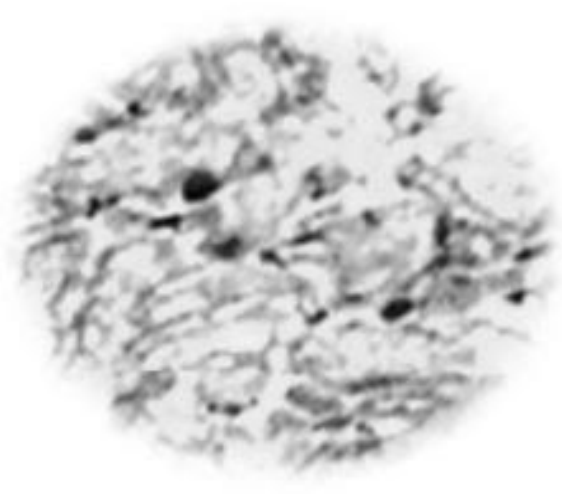

d

الشكل 10 الصور المجهرية بتكبيز X 200 لعينات من السبيكة 7075 معرضة للمعالجات الحرارية T6 وRRA قبل وبعد التشكيل.

\section{4. الاستنتاجات Conclusions}

1. حسنت المعالجات الحرارية بشكل عام الخواص الميكانيكية للسبيكة 7075 بنتيجة التغيرات الحاصلة في البنية البلورية و الحجم

البلوري والأطوار المترسبة بالتعتيق، وكذلك حسنت المعالجات الميكانيكية بالبثق الخواص الميكانيكية بنتيجة التصليد الانفعالي لبني الحاصل في بنية السبيكة 7075.

2. إن المعالجة الحرارية بالإرجاع وإعادة التعتيق RRA) Retrogression and re-ageing لسبيكة الألمنيوم 7075 حسنت الخواص الميكانيكية لها أكثر مما قد حسنته المعالجة الحرارية T6، حيث حصلنا على القيم العظمى لمقاومة الثند وإجهاد الخضوع و القساوة للسبيكة 7075 عند نطبيق المعالجة RRA، في حين كانت قيم هذه الخواص أقل عند تطبيق المعالجة

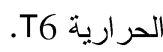

3. حسنت المعالجات الحرارية البنية المجهرية للسبيكة 7075 حيث أدت لانخفاض الحجم الحيبي، كما أدت لنقليل كثافة الثبكة النتعبية الموجودة في البنية، وقد كانت كثافة هذه النتعبات عند تطبيق المعالجة الحرارية RRA أقل منها عند تطبيث المعالجة الجية

\section{CONFLICT OF INTERESTS.}

- There are no conflicts of interest. 
References المصادر

[1] George E. Tot ten and D. Scott MacKenzie, Handbook of Aluminum Volume 7 Physical Metallurgy and Processes, MARCEL DEKKER, INC, 2003.

[2] TapanyUdomphol, Aluminum and its Alloy, Suranaree University of Technology, 2007.

[3] K. S. GHOSH and N. GAO, Transactions of Nonferrous Metals Society of China, vol. 21, 2011, p. 1199.

[4] F. Viana, A. M. P.Pinto, H. M.C. Santos and A. B. Lopes, "Retrogression and re-ageing of 7075 aluminium alloy microstructural characterization," Journal of Materials Processing Technology, no. $92 \pm 93$, p. $54 \pm 59,1999$.

[5] A. D. Isadare, B. Aremo, M. O. Adeoye, O. J. Olawale and M. D. Shittu, "Effect of Heat Treatment on Some Mechanical Properties of 7075 Aluminium Alloy," Materials Research, pp. 190-194, 2013.

[6] M. F. Ibrahim, A. M. Samuel, S. A. Alkahtani and F. H. Samuel, "A Novel Solution Heat Treatment Of 7075-Type Alloy," The Minerals Metals \& Materials Society, pp. 383-390, 2013.

[7] A.F. Oliveira, Jr., M.C. de Barros, K.R. Cardoso and D.N. Travessa, "Materials Science and Engineering A," The effect of RRA on the strength and SCC resistance on AA7050 and AA7150 aluminium alloys, no. 379, p. 321-326, 2004.

[8] Hong-ying LI, Jiao-jiao LIU, Wei-chen YU, Hui ZHAO and De-wang LI, "Microstructure evolution of $\mathrm{Al}-\mathrm{Zn}-\mathrm{Mg}-\mathrm{Cu}$ alloy during non-linear cooling process," Trans. Nonferrous Met. Soc. China, p. 1191-1200, 2016.

[9] P. K. Rout, M. M. Ghosh and K. S. Ghosh, "Microstructural, mechanical and electrochemical behavior of a $7017 \mathrm{Al}-\mathrm{Zn}-\mathrm{Mg}$ alloy of different tempers," Materials Characterization, 2015.

[10] CHEN Song-yi, CHEN Kang-hua, DONG Peng-xuan, YE Sheng-ping, HUANG Lan-ping and YANG Dai-jun, "Effect of a novel three-step aging on strength, stress corrosion cracking and microstructure of AA7085," J. Cent. South Univ, p. 1858-1862, 2016.

[11] M. Esmailian ‘M. Shakouri ‘A. Mottahedi g S. G. Shabestari" ‘Effect of T6 and Re-Aging Heat Treatment on Mechanical Properties of 7055 Aluminum Alloy "'International Journal of Chemical, Molecular, Nuclear, Materials and Metallurgical Engineering 'pp. 1291-1294 .2015 ،

[12] William F. Gale, Terry C. Totemeier, Smithells Metals Reference Book, 8 ed., ButterworthHeinemann, 2004. 